\title{
A new ultrasonic signal amplification method for detection of bacteria
}

\author{
Shiva Kant Shukla , Pablo Resa López , Carlos Sierra Sánchez , \\ José Urréjola and Luis Elvira Segura
}

\begin{abstract}
A new method is presented that increases the sensitivity of ultrasound-based techniques for detection of bacteria. The technique was developed for the detection of catalase-positive microorganisms. It uses a bubble trapping medium containing hydrogen peroxide that is mixed with the sample for microbiological evaluation. The enzyme catalase is present in catalase-positive bacteria, which induces a rapid hydrolysis of hydrogen peroxide, forming bubbles which remain in the medium. This reaction results in the amplification of the mechanical changes that the microorganisms produce in the medium. The effect can be detected by means of ultrasonic wave amplitude continuous measurement since the bubbles increase the ultrasonic attenuation significantly. It is shown that microorganism concentrations of the order of $10^{5}$ cells $\mathrm{ml}^{-1}$ can be detected using this method. This allows an improvement of three orders of magnitude in the ultrasonic detection threshold of microorganisms in conventional culture media, and is competitive with modern rapid microbiological methods. It can also be used for the characterization of the enzymatic activity.
\end{abstract}

Keywords: pulse-echo, rapid microorganism detection, catalase activity, bubble trapping medium

\section{Introduction}

The development of rapid methods for microorganism detection is a matter of great importance for different industrial sectors (for example, the food, pharmaceutical, water, biotechnology sectors) that deal with products that are susceptible to being contaminated. Contamination usually results in the spoilage of the product with obvious negative economical and environmental consequences. For this reason, reliable, simple, and rapid detection methods are in high demand. These techniques often play a key role in the quality control and decisively contribute to improve the production efficiency.

Although traditional methods such as $\mathrm{pH}$ measurements are still used for contamination detection in some industries, new technologies are changing the industrial microbiological quality control scenario. A great variety of new methods, for example, electric impedance measurements, ATP luminescence-based measurement, colorimetry, flow cytometry, epifluorescence, microcalorimetry, nucleic acid technologies, has been developed during the previous decades [1-3]. The reason for such diversity is the specific requirements, and the human and equipment resources available in each case. Different equipment will be used for microorganism quantification, identification, or sterility control. The number of samples required for analysis and the economic margins of the products will also have an impact on the technology employed. Therefore, different features, such as specificity, sensitivity, test rapidity, reliability, and equipment and consumable costs, have to be taken into account when choosing a suitable technique for the quality control of a given production process [4].

Among other physical quantities, living microorganisms are able to change the elastic properties of the medium in which they are present. This makes possible the use of ultrasonic methods to detect bacterial growth [5-7], albeit being less sensitive to microorganism concentration than other existing methods. The technique is competitive because it 
takes advantage of large sample incubation stages which are sometimes required. Nevertheless, if non-invasiveness is a less important criterion than sensitivity, a different strategy might be adopted. In this work the use of an especially designed medium to amplify ultrasonic changes produced by microorganisms is proposed. The technique reported here requires taking samples for testing and, therefore, it is invasive. However, it will be shown that ultrasonic characterization using such a medium provides a rapid and sensitive method for microbial detection.

In most of the bacteria detection methods found in the industry, the sample to be analyzed is previously prepared for measurement. For example, in electric impedance methods the sample is mixed with specific media, and the electric impedance changes as a consequence of the microbial metabolism. In the case of ATP-based methods, the sample liquid is solved in a medium containing reagents that react with ATP producing light, which is detected by an optical sensor. In a similar way, a specific medium suitable for microorganism detection using ultrasound is proposed and discussed here. This work explores the viability of reducing times and increasing the sensitivity needed for microbial detection in liquid using ultrasonic techniques. Even though it is invasive (it works by analyzing samples extracted from a pack or product), it still has attractive features from an industrial point of view: it is robust and can be easily scaled for multichannel equipment. In addition, the reagents used for the synthesis of the specific medium used (which constitutes the main economic waste in many quality evaluation techniques) may also be cost effective.

Following the same strategies as some biosensors [8] and impedance measuring devices [9], the idea of taking advantage of an enzymatic reaction as a method for signal amplification is translated to an ultrasonic method. In this case, the production of bubbles is pursued. Bubbles can be easily detected with acoustic methods because they produce significant changes in the mechanical properties of the medium (due to the large differences between the elastic characteristics of gases and liquids). Therefore, if a given medium reacts by producing bubbles when in contact with microorganisms, a significant increase in the sensitivity can be expected.

In order to analyze the efficiency of the method and the bacterial concentration thresholds that can be achieved following this approach, the hydrogen peroxide hydrolysis reaction induced by catalase was studied:

$$
2 \mathrm{H}_{2} \mathrm{O}_{2} \stackrel{\text { Catalase }}{\longrightarrow} 2 \mathrm{H}_{2} \mathrm{O}+\mathrm{O}_{2} .
$$

Therefore, if a sample contaminated with catalase-positive bacteria is placed in a medium containing hydrogen peroxide, bubbles appear when the oxygen concentration reaches the solubility limit in the measurement medium. In comparison with a conventional nutritive enriched medium, such a medium presents the drawback of being lethal for microorganisms, impeding them from multiplying. Nevertheless, the bubbles produced even at low microbial concentration make rapid ultrasonic detection possible.

To show that the detection effect is due to the hydrolysis induced by catalase, the ultrasonic method was first

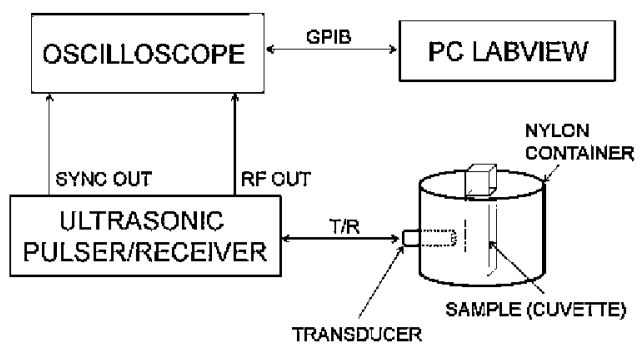

Figure 1. Experimental setup for ultrasonic measurements.

investigated using pure catalase, and then with the extracellular catalase that is present on some microorganisms. For microbial detection, which is the principal objective of the proposed technique, the method performance is evaluated from two points of view. Firstly, the improvements in detection time and sensitivity achieved with the proposed medium are compared with conventional media in order to determinate the sensitivity amplification obtained. Secondly, the performance of the proposed method was compared with a commercial microbial detection method based on ATP bioluminescence, which is a competitive technique since it also works with extracted samples.

\section{Material and methods}

\subsection{Ultrasonic signal amplification for microbial detection}

2.1.1. Ultrasonic measurement setup. A simple and robust ultrasonic measurement technique is presented based on a 'pulse-echo' configuration, which is widely used for non-destructive evaluation and characterization of materials. Ultrasonic pulses are emitted into the sample and on being reflected at the container walls the waves cross the sample again and impinge on the transducer. The transducer works as an emitting-receiving element. As shown in figure 1, the transducer is mounted horizontally in a partially water-filled nylon cylindrical container, which has an inner diameter of $17.5 \mathrm{~mm}$, a wall thickness of $7.0 \mathrm{~mm}$, and a height of $60 \mathrm{~mm}$. $3 \mathrm{ml}$ plastic commercial cuvettes (which are commonly used for spectrophotometric measurements) are placed inside and act as sample containers.

A $5 \mathrm{MHz}$ Panametrics A310S wideband transducer is driven by a Panametrics 5052UA ultrasonic pulser/receiver that emits $300 \mathrm{~V}$ spikes with a $5 \mathrm{kHz}$ repetition rate and also detects the incoming signal. This is acquired by a Tektronix TDS 2014 oscilloscope. The measurement process is computer-controlled using a software application programmed in LabView. It captures the traces acquired by the oscilloscope via a GPIB connection, performs the signal processing, displays the results in real time, and stores them.

The signal processing consists of wave amplitude evaluation. For this, the maximum values of the fast Fourier transform of acquired waveforms were calculated. Then, normalized amplitudes are obtained by dividing the amplitudes of successive acquisitions (one each $4 \mathrm{~s}$ ) by the amplitude measured at the beginning of each experiment. The evolution of this normalized amplitude provides a measurement of the changes produced in the medium due to bubble proliferation as the indicator of microbial presence. 
2.1.2. Specific medium for ultrasonic signal amplification. The analysis medium for the ultrasonic test is a matter of chief importance in obtaining good performance. Three requirements must be met: (i) stability in the presence of hydrogen peroxide, which is a highly reactive molecule, (ii) the ability to trap the bubbles formed, and (iii) having sufficient fluid to allow rapid mixing of the hydrogen peroxide solution.

The selected medium was based on American Bacteriological Agar (cat. 1802.00; CONDA). A 1\% w/w suspension of agar in distilled water was made and autoclaved at $120^{\circ} \mathrm{C}$ to avoid cross contamination. It was cooled to room temperature inside an ultra violet (UV) flow chamber.

The test was performed as follows. $1 \mathrm{ml}$ of the agar medium is mixed with $1 \mathrm{ml}$ of the sample and analyzed in the $3 \mathrm{ml}$ plastic cuvette. Then $100 \mu \mathrm{l}$ of hydrogen peroxide solution $10 \mathrm{M}$ in distilled water is added and shaken three times vigorously to ensure good homogenization of the peroxide. The cuvette is then immediately placed inside the nylon container for ultrasonic continuous monitoring.

Additionally, the testing medium used is simple, consisting of agar, water and hydrogen peroxide. It is economically competitive in comparison with other media based on optical markers (for example, luminescenceand fluorescence-based methods) that are relatively more expensive.

\subsubsection{Amplitude measurement calibration. The oscilloscope} has an amplitude precision of 8 bits that allows relative changes of less than $1 \%$ to be detected, assuming that the initial wave scales at half the maximum scale. However, other factors have greater significance in the accuracy of the microorganism detection.

There are two important factors which influence the stability of blank measurements. The first one is the temperature stability ( $\pm 2{ }^{\circ} \mathrm{C}$ for rapid measurements), and the second one is the stability of the medium used for measurement. The experimental technique was checked for two reference media: distilled water and the agar medium described in the previous section. Figure 2 shows the ultrasonic wave amplitude evolution obtained for both media. Temperature fluctuations do not produce changes greater than $2 \%$ in water and therefore, the greater amplitude variations found in the developed medium are mainly due to the instabilities of this complex organic material. Nevertheless changes smaller than $5 \%$ were obtained through all the measurements.

There may be other error sources affecting microbial detection measurements, such as random bubble nucleation (implying a differing bubble count or movement in front of the acoustic transmission path between different measurements), and the variability of the biological assays (which depend on many factors: microorganism stress, different strains used, final concentration reached after dilution, etc). This reduction in repeatability may cause variations in the time decay obtained in each experiment. For this reason five experiments were performed for each microbial concentration and strain and the average calculated.

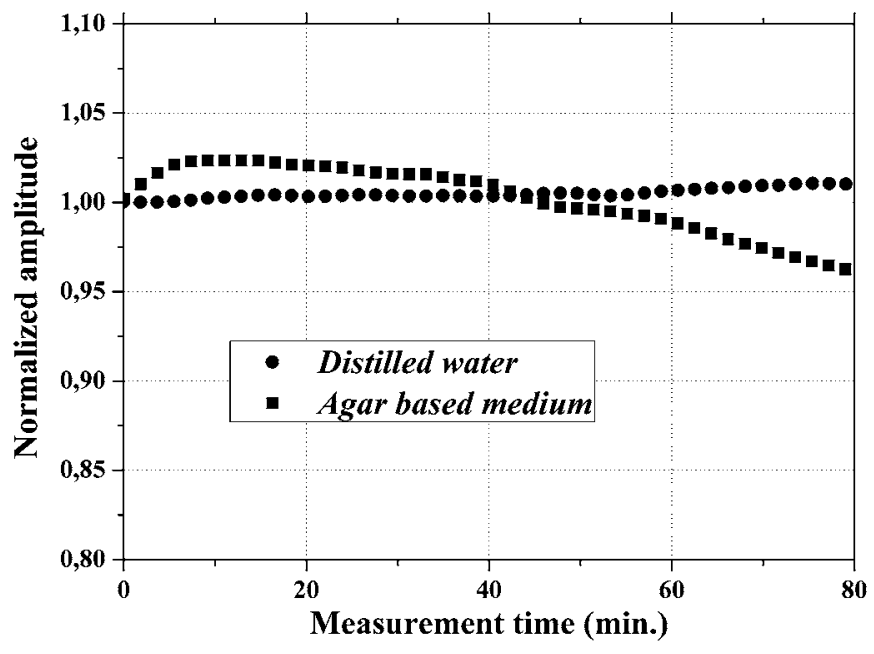

Figure 2. Evolution of ultrasonic amplitude for distilled water (circles) and the developed agar-based medium (squares).

\subsection{Reference experimental measuring devices}

2.2.1. Catalase activity characterization. Catalase activity is the basic biochemical reaction employed here. Spectrophotometric assays were performed in order to relate the activity of the pure enzyme with that of bacterial enzymes. A UV-mini 1240 Spectrophotometer (Shimadzu) was used for this purpose.

A catalase solution (Sigma, C1345 from bovine liver) was prepared in a potassium phosphate buffer ( $\mathrm{pH} 7$ ) with a concentration of $0.025 \mathrm{~g} \mathrm{l}^{-1}$. Another solution of $0.036 \%$ $\mathrm{w} / \mathrm{w}$ hydrogen peroxide $(50 \%$ hydrogen peroxide solution in water, 516813, Sigma Aldrich) was also prepared using the same buffer as a solvent. By mixing them in a $3 \mathrm{ml}$ spectrophotometric cuvette, a $34.5 \% \mathrm{v} / \mathrm{v}$ concentration of catalase solution in hydrogen peroxide solution results and the evolution of the solution optical absorbance at $240 \mathrm{~nm}$ is monitored. The initial decrease rate of the absorbance characterizes the enzymatic activity. Following the suppliers' recommendations [11], the formula used to calculate the activity is $[11,12]$

$$
\text { Enzyme (units } \mathrm{ml}^{-1} \text { ) }=\frac{34.5}{t} \text {. }
$$

$t$ is the time in minutes required for the absorbance at $240 \mathrm{~nm}$ to decrease from 0.45 to 0.40 units which corresponds to a change in concentration from 10.3 to $9.2 \mathrm{mM}$.

Nevertheless, the experimental method accuracy improves when the data are fitted to an exponential-decay function using data from the beginning of the reaction. The slope is obtained after the first reaction minute to avoid deviation coming from reagent mixing. From this slope, $s$ (absorbance units $\min ^{-1}$ ), the activity can be obtained as

$$
\text { Enzyme (units } \mathrm{ml}^{-1} \text { ) }=s \cdot \frac{34.5}{0.05}=690 \cdot s .
$$

This is equivalent to equation (2), but using exponential instead of linear behavior.

The solvent Luria-Bertani (LB) broth [13], instead of the phosphate buffer, was used when analyzing the catalase activity of microorganisms. The former is a nutritionally 
rich medium often used to culture microorganisms, and has a higher initial absorbance having values between 1.10 and 0.50 absorbance units depending on the specific microorganism concentration. Although the LB broth has an initial slightly higher $\mathrm{pH}$ value (7.5), this does not alter the catalase activity [14], and hence the same equation (3) (which does not depend on the initial absorbance value) can be applied in order to calculate the enzymatic activity.

\subsubsection{Ultrasonic microbial detection using conventional} nutritive media. Ultrasound microbial detection using conventional nutritive media is based on durable changes produced during microbial growth. In these media, largescale bubble formation does not typically occur and sound speed measurements using a thermally controlled ultrasonic device are better suited for detection purposes. In order to measure propagation times and concentration thresholds in these conventional media, a different instrument from that described in section 2.2.1 was used (a Milisound device, described in detail in [10]).

\subsubsection{Microbial detection using a commercial instrument based on bioluminescence induced by ATP. Biolumines- cence induced by ATP is becoming one of the most important industry methods for microbial detection. For this reason a comparison was made of the microbial detection thresholds and detection times achieved with the proposed ultrasonic technique. For this purpose a Promilite III (Promicol) was used as a reference method for microbial sample analysis.}

\subsection{Bacterial strains and microorganisms}

Three catalase-positive microorganisms Escherichia coli, Bacillus amyloliquefaciens, and Pseudomona putida were selected for this study. All of them were provided by the Environmental Microbiology Group (CIB, CSIC). The DH5 $\alpha$ strain of $E$. coli comes from 'Invitrogen' USA [13]. The other strains 'ATCC 23842' [15] and 'KT2442' [16] were supplied by the Spanish Type Culture Collection (CECT) and the University of Valencia, respectively.

Bacterial cells were grown overnight in LB at $37^{\circ} \mathrm{C}$ in an orbital incubator. The initial concentration of bacterial cells was determined by spectrophotometric measurements assuming that 1 unit of optical density $\left(\mathrm{OD}_{600 \mathrm{~nm}}\right)$ corresponds to approximately $8.1 \times 10^{8}$ cells $\mathrm{ml}^{-1}$ [13]. Different concentrations of microorganisms ranging from $10^{8}$ to $10^{5}$ cells $\mathrm{ml}^{-1}$ were obtained by dilution with a saline solution (sodium chloride, $0.99 \% \mathrm{w} / \mathrm{w}, \mathrm{S} 3014$; Sigma) for use with the ultrasonic tests, and by dilution with LB broth for the ATP tests carried out in an UV laminar flow chamber.

\section{Results and discussion}

\subsection{Ultrasonic detection thresholds for conventional nutritive media}

The growth of the selected strains in the rich LB medium was monitored by ultrasound using the aforementioned Milisound

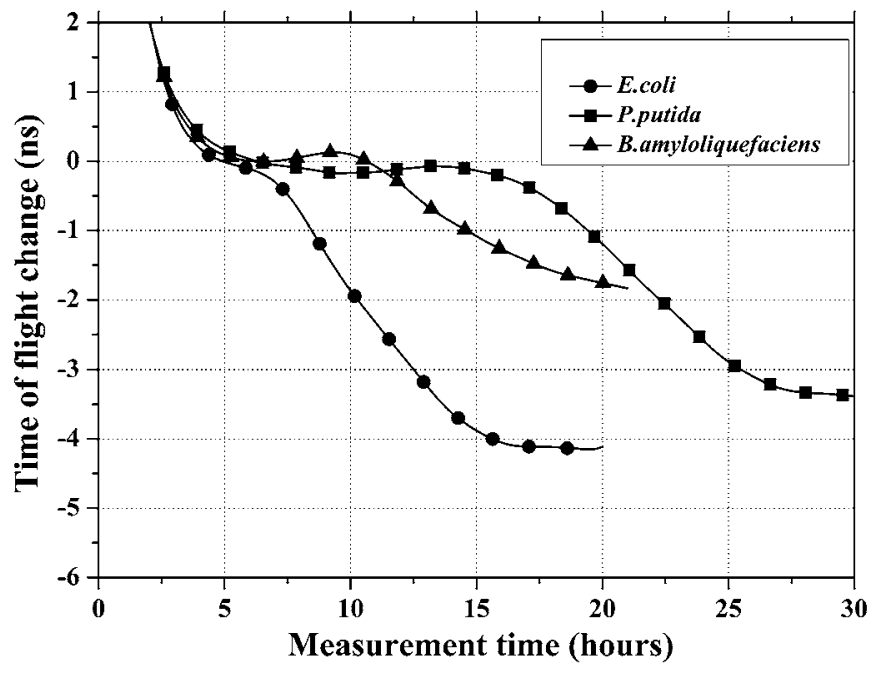

Figure 3. TOF changes during the growth of different bacteria using a conventional $\mathrm{LB}$ medium at $37^{\circ} \mathrm{C}$.

Table 1. Concentration thresholds for different microorganisms obtained using a conventional ultrasonic monitoring technique.

\begin{tabular}{ll}
\hline Microorganism & $\begin{array}{l}\text { Concentration } \\
\left(\text { cells } l^{-1}\right)\end{array}$ \\
\hline E. coli & $3 \times 10^{8}$ \\
P. putida & $8 \times 10^{7}$ \\
B. amyloliquefaciens & $7 \times 10^{7}$ \\
\hline
\end{tabular}

equipment [10] at $37^{\circ} \mathrm{C}$ incubation temperature. A sample of overnight cultured bacteria in LB broth was diluted in LB to obtain a concentration of $10^{5}$ cells $\mathrm{ml}^{-1} .1 \mathrm{ml}$ of this medium for each strain was inoculated in stirred closed bottles containing $100 \mathrm{ml}$ of the same culture medium. The initial concentration of bacteria after this inoculation was $10^{3}$ cells $\mathrm{ml}^{-1}$. The decrease in time of flight (TOF) during the first 4 to $5 \mathrm{~h}$ period is due to thermal changes in the medium. This decay in TOF ends when the cultures reach the equilibrium temperature set and a 'plateau' follows in the TOF which corresponds to the latency stage of microorganisms and the beginning of the growing phase where the bacterial growth still does not result in any detectable change using the ultrasonic measurement. It can be seen that after these plateaus the three strains produce decay in TOF. This is detectable first in $E$. coli, and last in $P$. putida (see figure 3).

After the latency stage, a TOF drop of $1 \mathrm{~ns} \mathrm{~h}^{-1}$ is taken as an indication of bacterial growth. The spectrophotometric measurement from a sample taken at this point reveals the bacterial concentration threshold for the ultrasonic technique in the LB medium. The results are shown in table 1. An average value of about $10^{8}$ cells $\mathrm{ml}^{-1}$ was obtained for the selected strains. These are in the same order as the values given in [6], for microorganism detection in milk. Therefore, if signal amplification could be successfully achieved by the technique proposed in this paper, detection thresholds should be diminished from $10^{8}$ cells $\mathrm{ml}^{-1}$ and detection times should be decreased from $4 \mathrm{~h}$, which is the minimum stabilization time required. 


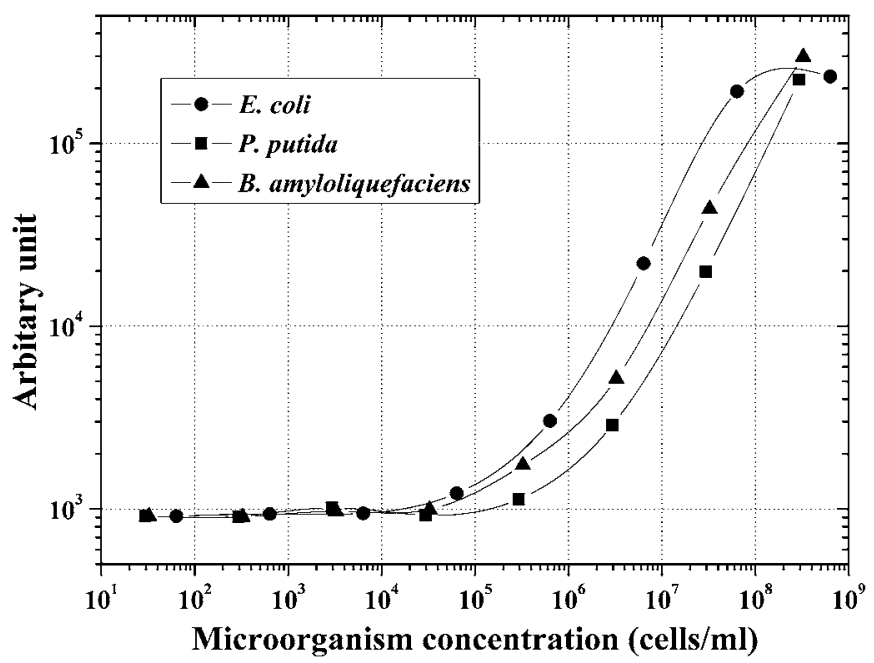

Figure 4. Detection of bacteria using ATP bioluminescence.

\subsection{ATP detection}

An ATP-bioluminiscence method was used to assess the efficiency of standard industrial methods for detecting microorganisms. Two different cultures of each of the three strains described above were prepared as described in section 2.3. Dilutions of the six cultures were performed until undetectable bacterial ranges were reached. Blank tests of sterile LB broth were also performed, and a medium value of 940 arbitrary units (A.U.) was obtained which was coherent with the values obtained in the most diluted bacterial samples. This blank-test reading takes into account free ATP present in the LB. $50 \mu \mathrm{l}$ samples of each dilution and blanks were put in a 96 well microplate for the ATP analysis. Afterwards the luminescence reading was taken.

Both cultures belonging to the same strains gave similar results, and their average was calculated and shown in figure 4. It can be seen that for B. amyloliquefaciens and E. coli, detection ranges of the order of $10^{5}$ cells $\mathrm{ml}^{-1}$ were reached and somewhat higher values, between $10^{5}$ and $10^{6}$ cells $\mathrm{ml}^{-1}$, were obtained for the $P$. putida tests. Measurement time is on the order of $5 \mathrm{~min}$ for these analyses. These are the concentration thresholds and time scales which might be required in order to make a microorganism detection technique competitive with current techniques.

\subsection{Detection of catalase using ultrasound}

Pure catalase experiments at room temperature were carried out in order to study the performance of the ultrasonic measuring method. The catalase activity was previously assessed following section 2.2.1.After mixing the catalase solution with the agar, hydrogen peroxide was added and the variation of the normalized ultrasonic wave amplitude evolution was measured and registered. In figure 5, a measurement obtained with 1 unit $\mathrm{ml}^{-1}$ of catalase is presented. Due to the presence of catalase, the hydrolysis of peroxide molecules initiates bubble production. These bubbles are trapped in the agar gel medium and increase the attenuation of ultrasound. Figure 5 shows clearly that the normalized

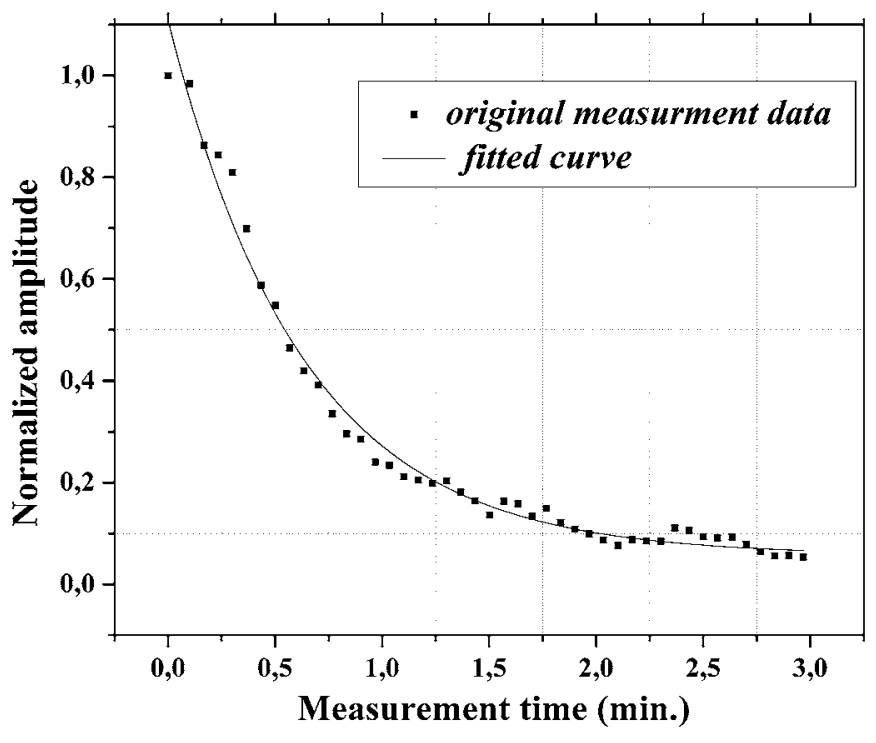

Figure 5. Evolution of ultrasonic amplitude during hydrogen

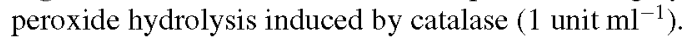

amplitude diminishes with time and follows an exponentialdecay trend:

$$
y=\mathrm{e}^{-\frac{x}{k}}
$$

The experimental data fit of this function is plotted as a continuous line in figure 5. Small bubble movement, coalescence and size changes produce deviations from the expected theoretical behavior. Nevertheless, the decay constant, $k$, obtained from these curves, can be used to characterize the enzymatic activity.

The catalase solution is diluted to reach activities from 1 to $10^{-3}$ units $\mathrm{ml}^{-1}$. A blank test is also performed (adding a phosphate buffer solution with no catalase) to ensure that no hydrogen peroxide hydrolysis is produced by cross contamination or by other reagents. Five experiments were performed for each dilution, and an average decay constant was obtained. The constant was corrected with the effect of the medium change shown in figure 2 . The decay constants are shown in figure 6 and error bars are also given.

From this figure, it can be seen that there is a nonlinear relationship between the enzymatic activity and the (initialrate) decay constant. This is thought to be due to the fact that ultrasonic attenuation for bubble suspensions is not only a function of the concentration volume of bubbles but also of the bubble size [17]. For a $5 \mathrm{MHz}$ wave, there exists a bubble size that will give the maximum wave attenuation. For water, which is elastically close to the $1 \%$ agar suspension, the resonant diameter is in the order of $1 \mu \mathrm{m}$. During the experiments, the bubbles produced are quickly and readily visible and, therefore, above this resonance. When bubbles appear and begin to grow, two different effects compete with respect to the ultrasonic propagation: (i) the increase in the oxygen volume concentration (which produces increasing attenuation), and (ii) the increase in bubble size (which produces for sizes after the resonance a decreasing attenuation). For low gas concentrations, the latter compensates for the decrease in amplitude due to bubble formation. 


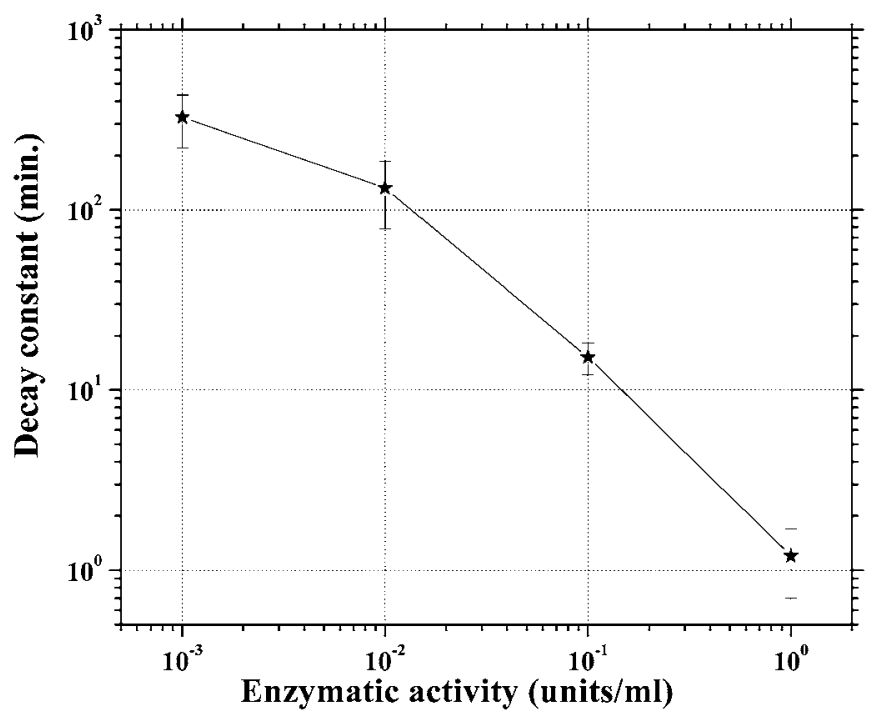

Figure 6. Ultrasonic amplitude decay constant as a function of enzymatic activity.

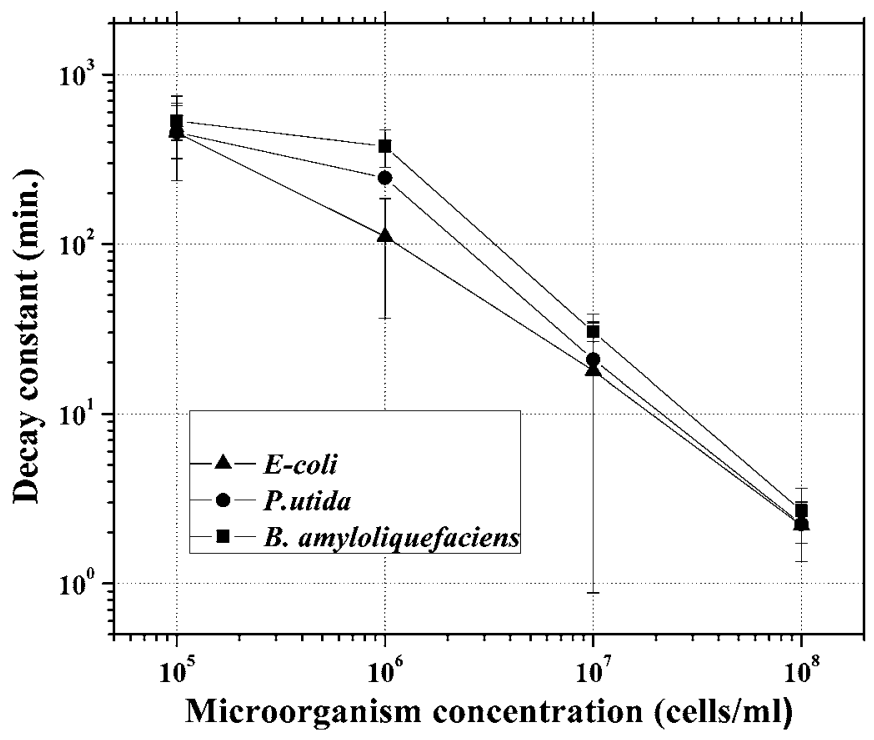

Figure 7. Ultrasonic amplitude decay constant as a function of the microorganism concentration.

\subsection{Rapid detection of catalase-positive bacteria using ultrasound}

In a similar way, ultrasonic experiments were performed for the abovementioned catalase-positive microorganisms E. coli, $P$. putida and B. amyloliquefaciens. Different microbial concentrations $\left(10^{5}, 10^{6}, 10^{7}\right.$ and $10^{8}$ cells $\left.\mathrm{ml}^{-1}\right)$ were analyzed at room temperature, and the wave amplitude decrement was analyzed. Blank experiments were also performed, as in the case of pure enzymatic assays. Again, five experiments were performed for each dilution and strain, and an average decay constant was obtained. This is plotted as a function of the microbial concentration in figure 7 .

After a large number of measurements, a relationship was always seen between a 10\% amplitude decrement and reaction being detected. Therefore, none of the experimental variability sources could explain changes greater than this $10 \%$. This threshold can be considered the minimum amplitude needed for detection.

Detections are achieved for all the strains tested at all the concentrations analyzed (from $10^{5}$ up to $10^{8}$ cells $\mathrm{ml}^{-1}$ ). Nevertheless, the measurement time needed depends on the microbial charge. The highest decay constant obtained was $380 \mathrm{~min}$ for $10^{5}$ cells $\mathrm{ml}^{-1}$ of $B$. amyloliquefaciens. With this decay constant, a decrement of $10 \%$ in amplitude requires about 40 min from the beginning of the assay.

The nonlinear behavior of the decay constant with the microorganism concentration is similar to that obtained in the pure enzymatic experiments. It can therefore be concluded that the measurements obtained with microorganisms respond to the same induced process using pure catalase.

One of the main objectives of this work is to improve the sensitivity of ultrasound propagation measurement so as to exploit this technique as a microbiological rapid detection method. Comparing the measurements shown in sections 3.1 and 3.4, microorganism concentration thresholds were improved by three orders of magnitude with the enzymatic method proposed, which is a significant result. The continuous measurement method described in section 3.1 is based on microbial growth and therefore it needs long-duration tests in order to achieve detectable levels. Minimum detection times of $4 \mathrm{~h}$ were needed because sample thermal stabilization is mandatory. The method proposed in this work operates directly on the sample and no growth is required. The detection times obtained were also considerably reduced, and can be expressed in minutes (from less than $1 \mathrm{~min}$ for $10^{8} \mathrm{cfu} \mathrm{ml}^{-1}$ to $40 \mathrm{~min}$ for $10^{5} \mathrm{cfu} \mathrm{ml}^{-1}$ ).

If the detection thresholds are compared with an efficient and well-established method such as ATP bioluminiscence, similar levels are obtained, although times are somewhat better in this last case. These results open up the possibility of commercializing the proposed method as a rapid technique for microbial detection. Development of media and wave treatment techniques could probably further improve the sensitivity obtained through this work and the price of the reagents would be clearly lower than those used in the ATP technique.

Another important issue for discussion is the potential performance of the ultrasonic method as an enzymatic characterization technique. Following the conventional spectrophotometric method described in section 2.2.1 (see figure 8 ), the catalase activity of the strains was analyzed.

Time decay constants obtained in these experiments for microbial concentrations of the order of $10^{8}$ cells $\mathrm{ml}^{-1}$ were higher than $1000 \mathrm{~min}$, which is three orders of magnitude higher than that given by the ultrasonic method proposed. This means that the ultrasonic method is better suited to characterize low enzymatic concentrations. 0.05 units $\mathrm{ml}^{-1}$ can be considered as the minimum enzymatic activity for detecting catalase using spectrophotometric methods [18]. This limit can be decreased down to 0.001 units $\mathrm{ml}^{-1}$ by using ultrasound. In terms of linearity, the spectrophotometric method seems to be advantageous although it is not completely linear either, as shown in figure 8 , especially for the case of the $P$. putida assays. 


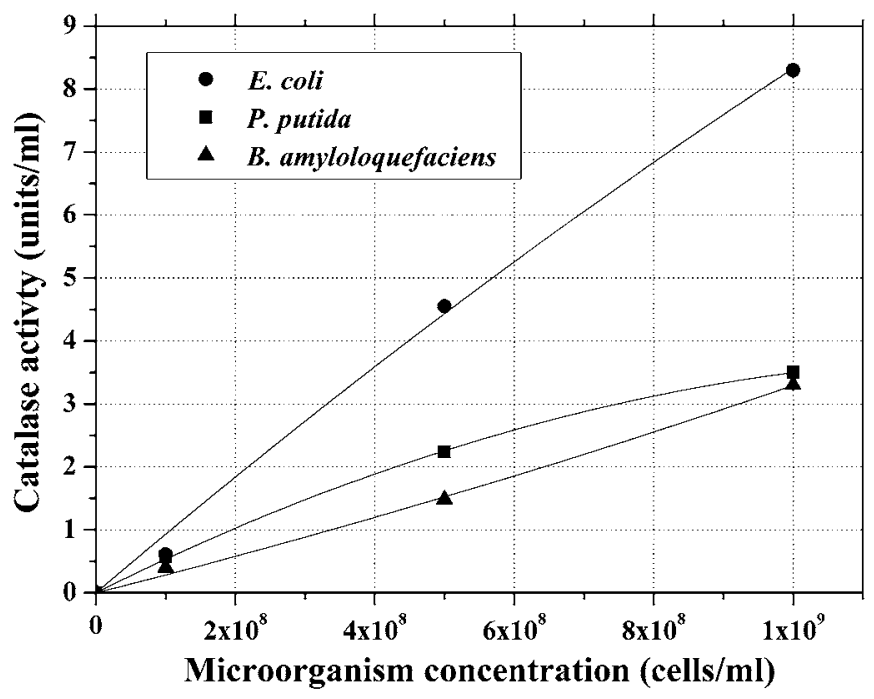

Figure 8. Catalase activity of the microbial strains analyzed as a function of the concentration by spectrophotometry.

Table 2. Coefficients obtained from the second-order fitting of the activity versus microbial concentration curve of figure 8 .

\begin{tabular}{llc}
\hline Microorganism & $b$ (units/cells) & $c$ (units ml/cells $\left.{ }^{2}\right)$ \\
\hline E. coli & $9.4 \times 10^{-9}$ & $-1.1 \times 10^{-18}$ \\
P. putida & $5.5 \times 10^{-9}$ & $-2.0 \times 10^{-18}$ \\
B. amyloliquefaciens & $2.8 \times 10^{-9}$ & $4.9 \times 10^{-19}$ \\
\hline
\end{tabular}

Finally, the possible influence of any other mechanism affecting the ultrasound amplitude evolution in the same way as the wave disturbance produced by the microorganism presence or the LB broth is analyzed by comparing the ultrasonic experiments carried out with pure catalase and positive catalase bacteria. It is clear that the trend of the curves shown in figures 6 and 7 is the same, although in order to perform this on a quantitative basis, the concentration of microorganisms would need to be translated to enzymatic activity. For this, the activity of microbial solutions at low concentration is obtained from a second-order polynomial fitting of figure 8 (also shown in this figure as solid lines) where the independent term is set to zero because the lack of microorganisms must give null activity. The second-order coefficient takes into account nonlinear behavior as a result of activity inhibition by excess of enzyme [19]. Then, the fitting polynomial is

$$
y=b x+c x^{2} .
$$

The values of coefficients ' $b$ ' and ' $c$ ' for different microorganisms are given in table 2.

Once the relation between microbial concentration and activity is calculated, ultrasonic decay constants from both the pure enzyme and microbial solutions can be plotted (figure 9). Good agreement is obtained. These results show that the ultrasonic method can be used not only to detect microbial contaminations but also to characterize enzymatic reactions induced by these microorganisms.

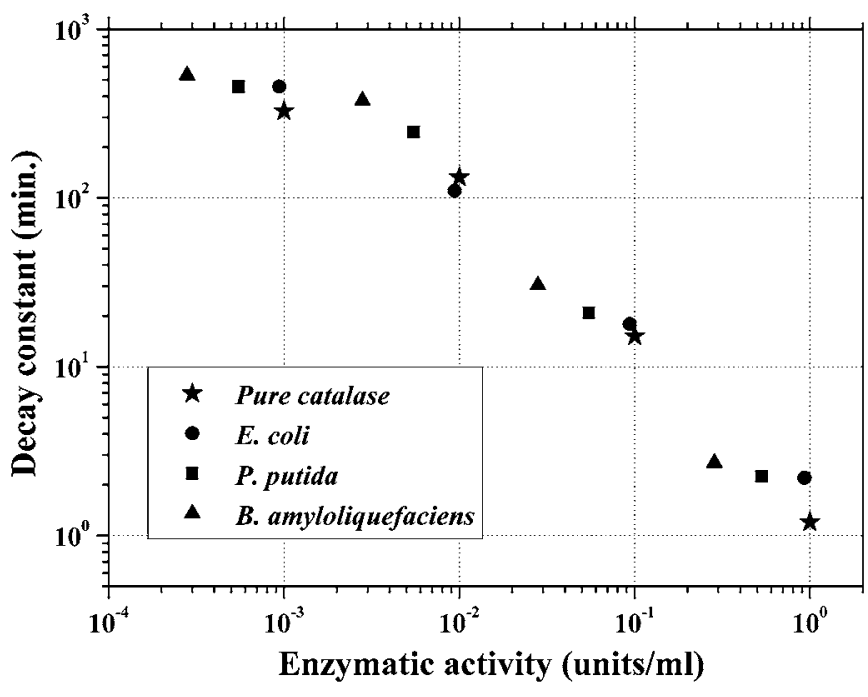

Figure 9. Decay constant as a function of the enzymatic activity of pure catalase and different analyzed microorganisms.

\section{Conclusions}

Using special media for microorganism detection improvements is commonplace (for example, optical markers in spectrophotometry or fluorometry, electrolytic media for impedancimetry). These markers amplify the signal for microbiological detection. A new procedure for amplifying the acoustic signal produced by the presence of microorganisms when using ultrasonic evaluation techniques is proposed in this work. The novel element relies on taking advantage of enzymatic reactions induced by these microorganisms to produce bubbles and amplify the ultrasonic response. In practice, the concept was applied to the detection of catalase-positive microorganisms released in a medium containing hydrogen peroxide. It was shown that this technique gives a noticeable improvement of the microorganism detection thresholds, which were decreased by three orders of magnitude, and a reduction of detection times from hours to minutes. As far as the authors are aware, this is currently the best sensitivity obtained for bacterial detection using ultrasonic wave propagation methods. This sensitivity is similar to that reached by ATP bioluminescence, which is a well-established method and commonly employed in the industry. The method can be easily implemented in a robust and friendly device, allowing a cost-effective and rapid microbiological evaluation of industrial samples.

The study was completed by carrying out pure catalase experiments. Spectrophotometric and ultrasonic methods were found to be completely coherent, showing that ultrasound can be applied for the characterization of enzymatic reactions. Moreover, the ultrasonic method is better suited for catalase characterization when low amount of enzyme is present.

From this work two research lines are opened with the goal of improving the method. The first is to study the performance of other ultrasonic parameters (for example, sound speed, scattering coefficient) and operation at different frequencies. The second is to analyze other reaction types induced by microorganisms with bubble production. 


\section{Acknowledgments}

This work was funded by the Spanish Ministry of Science and Innovation (CICYT DPI2007-61759, DPI2010-17716) and the JAE-CSIC Predoctoral Program/European Social Fund (JAE-PreDoc2009). The authors wish to thank the group of Environmental Microbiology from the CIB, CSIC, for the bacterial strains provided and Dr David K Anthony for paper review.

\section{References}

[1] Fung D 2008 Rapid methods and automation in food microbiology: 25 years of development and predictions ARI: Bull. Istanbul Technical Univ. 54 (4) 45-55

[2] Pishawikar M S, Singhal R S and Kulkarni P R 1992 Rapid non-microbiological methods for detecting microorganisms in foods Trends Food Sci. Technol. 3 165-9

[3] Vanne L, Karwoski M, Karppinen S and Sjiiberg A-M 1996 HACCP-based food quality control and rapid detection methods for microorganisms Food Control 7 216-63

[4] Kress-Rogers E and Brimelow C J C 2001 Instrumentation and Sensors for the Food Industry 2nd edn (Cambridge: Woodhead Publishing)

[5] Hæggström E 1997 Ultrasonic detection of microbe contamination in premade food Acta Polytech. Scand. Appl. Phys. 214 1-115

[6] Elvira L, Sampedro L, Matesanz J, Gómez-Ullate Y, Resa P, Iglesias J R, Echevarría F J and Montero de Espinosa F 2005 Non-invasive and non-destructive ultrasonic technique for the detection of microbial contamination in packed UHT milk Food Res. Int. 38 631-8

[7] Sierra C, Elvira L, Garcia L, Resa P and Galán B 2009 Monitoring Escherichia coli in M63 media by ultrasonic noninvasive methods and correlation with spectrophotometric and HPLC technique Appl. Microbiol. Biotechnol. 85 813-21

[8] Serra B, Zhang J, Morales M D, Guzmán-Vázquez de Prada A, Reviejo A J and Pingarrón J M 2008 A rapid method for detection of catalase-positive and catalase-negative bacteria based on monitoring of hydrogen peroxide evolution at a composite peroxidase biosensor Talanta 75 1134-9

[9] Yang L and Bashir R 2008 Electrical/electrochemical impedance for rapid detection of food borne pathogenic bacteria Biotechnol. Adv. 26 135-50

[10] Elvira L, Durán C, Sierra C, Resa P and Montero de Espinosa F 2007 Ultrasonic measurement device for the characterization of microbiological and biochemical processes in liquid media Meas. Sci. Technol. 18 2189-96

[11] Beers R F Jr and Sizer I W 1952 A spectrophotometric method for measuring the breakdown of hydrogen peroxide by catalase J. Biol. Chem. $195133-40$

[12] Stern K G 1937 On the absorption spectrum of catalase J. Biol. Chem. 121 561-72

[13] Sambrook J and Russell D W 2001 Molecular Cloning: A Laboratory Manual 3rd edn (New York: Cold Spring Harbor Laboratory Press)

[14] Aebi H 1984 Catalase in vitro Methods Enzymol. 105 121-6

[15] Priest F G, Goodfellow M, Shute L A and Berkeley R C W 1987 Bacillus amiloliquefaciens sp. nov. nom. rev. Int. J. System. Bacteriol. 37 69-71

[16] Bagdasarian M, Lurz R, Ruchert B, Frankli F C H, Bagdasarian M M, Frey J and Timmis K N 1981 Specific-purpose plasmid cloning vectors: II. Broad host range, high copy number, RSF1010-derived vectors for gene cloning in Pseudomonas Gene 16 237-47

[17] Temkin S 2000 Attenuation and dispersion of sound in dilute suspensions of spherical particles J. Acoust. Soc. Am. $108126-46$

[18] Li Y and Schellhorn H E 2007 Rapid kinetic microassay for catalase activity J. Biomol. Tech. 18 (4) 185-7

[19] Vasudevan P T and Weiland R H 1990 Deactivation of catalase by hydrogen peroxide Biotechnol. Bioeng. 36 788-9 